\title{
DISKUSSION
}

DDS - Die Deutsche Schule

112. Jahrgang 2020, Heft 2, S. 245-248

https://doi.org/10.31244/dds.2020.02.10

(C) 2020 Waxmann

Isabell van Ackeren, Manuela Endberg \& Oliver Locker-Grütjen

\section{Chancenausgleich in der Corona-Krise: Die soziale Bildungsschere wieder schließen}

\section{Zusammenfassung}

Die aktuelle Corona-Krise verschärft mit jedem Tag ohne Schule vor Ort bestehende soziale Ungleichheit. Dabei spielt auch die sogenannte Digitale Kluft eine Rolle. Entsprechende Erkenntnisse aus vorliegender Forschung unterstützen die Sorge der sich weitenden Bildungsschere. Wenn Schulen sukzessive wieder geöfnet werden, sollten Kinder und Jugendliche aus weniger privilegierten Milieus besonders berücksichtigt werden. Unter der Perspektive von Bildungsgerechtigkeit kann hier Bildungspolitik unseres Erachtens unkonventionell und schnell handeln, auch unter breiterer Einbindung von Lehramtsstudierenden.

Schlüsselwörter: Ausgleich von Bildungschancen, Bildungsgerechtigkeit, Digitale Kluft

\section{Compensation of Unequal Educational Opportunities in the Corona Crisis: Closing the Social Education Gap again}

\section{Abstract}

The current corona crisis aggravates existing social inequality with every day without schooling on-site. Here the so-called digital divide also plays a crucial role. Corresponding findings from existing research reinforce the concern about the widening education gap. When schools are gradually reopening, children and young people from less privileged milieus should be given special consideration. From the perspective of educational justice, we believe that education policy can act unconventionally and quickly here, also with the broader involvement of teacher training students.

Keywords: compensation of unequal educational opportunities, educational justice, digital divide

Dass es in Deutschland eine besonders enge Kopplung zwischen sozialer Herkunft und schulischen Leistungen gibt, ist seit Jahrzehnten empirisch belegt. Dieser Zusammenhang konnte bis heute nicht systematisch aufgebrochen werden - und wird 
durch die Corona-Krise massiv verschärft, wenn Schule insbesondere für die Gruppe der Kinder als physischer Ort nicht zur Verfügung steht, die schon vor der Krise im Bildungssystem abgehängt war. Unterschiede im Zugang zu und im Umgang mit digitalen Medien verstärken die Ungleichheit. Hierfür gibt es mehrere, empirisch belegte Hinweise, die zeigen, dass politisch schnell gehandelt werden sollte, wenn es jetzt zu einer schrittweisen Öffnung von Schule kommt. Drei ausgewählte Argumentationslinien:

\section{Probleme längerer Zeiträume ohne Beschulung}

Es gibt eine eindeutige Forschungslage, dass lange Phasen ohne Schulen - etwa in den Sommerferien - bei Kindern aus benachteiligten Verhältnissen zu Rückschritten beim Kompetenzerwerb führen, im Unterschied $\mathrm{zu}$ Kindern aus privilegierten Familien mit viel mehr Unterstützungsmöglichkeiten. Wie alle Familien mit schulpflichtigen Kindern mit der aktuellen Lage umgehen (können), dürfte höchst unterschiedlich aussehen: von vielfältigen, lernrelevanten, sprachfördernden und die Kinder auch emotional stärkenden Interaktionen mit allen technischen Möglichkeiten bis hin zu Situationen, in denen sie vollständig den Medien überlassen werden (sofern diese überhaupt verfügbar sind) oder sogar häuslicher Gewalt ausgesetzt sind und an Lernen nicht zu denken ist. Je länger solche Phasen andauern, desto größer sind die damit verbundenen Nachteile.

\section{Unterschiedlicher Zugang zu digitalen Medien}

Studien zeigen weiterhin, dass es Familien gibt, die komplett ohne Internetanschluss, Computer und Drucker leben und die nicht ständig Zugriff auf digitale Endgeräte haben. Dies ist eine Facette des digital gap, der digitalen Kluft innerhalb unserer Gesellschaft. Somit kann es Probleme geben, an die schulischen Lernaufgaben heranzukommen und diese in der vorgegebenen Frist $\mathrm{zu}$ bearbeiten. Familiäre Streitigkeiten sind dabei nicht ausgeschlossen, insbesondere wenn es keinen Freiraum gibt, Familienmitglieder auf engstem Raum zusammenleben und es Existenzängste gibt. Auch aus diesen Gründen fällt der Zugang zu Bildung, die derzeit weitgehend der Sphäre der Familie überlassen bleibt, sozial ungleich aus.

\section{Fehlende Kompetenzen im Umgang mit digitalen Tools}

Die International Computer and International Literacy Study (ICILS) hat 2013 erstmalig die digitalen Kompetenzen von Achtklässler*innen im internationalen Vergleich getestet. Dabei ist Deutschland im Mittelfeld gelandet. Besonders besorgniserregend: Ca. ein Drittel der Schüler*innen verfügte nur über sehr rudimentä- 
re Kompetenzen im Umgang mit digitalen Medien (z.B. einen als solchen gekennzeichneten Hyperlink öffnen, eine digitale Datei nach entsprechender Erklärung speichern). Der Anteil der Schüler*innen aus sozial benachteiligten Familien an diesem Drittel fiel dabei besonders hoch aus. Fünf Jahre später konnten in der zweiten Durchführung von ICILS die genannten Ergebnisse nahezu exakt repliziert werden, wobei bildungspolitische Maßnahmen, wie der DigitalPakt Schule, noch nicht wirken konnten. Aber man muss zur Kenntnis nehmen, dass es derzeit eine Situation gibt, in der der Zugang zu und der kompetente Umgang mit digitalen Medien sehr ungleich verteilt sind. Dabei ist auch klar, dass die Lehrkräfte selbst überwiegend noch nicht hinreichend für den Umgang mit und den sinnvollen Einsatz von digitalen Medien ausgebildet sind, insbesondere auch im Hinblick auf die skizzierten heterogenen Lernausgangslagen. Viele digitale Tools sind auch noch nicht wirklich qualitätsgeprüft bzw. nicht speziell für den Schulkontext entwickelt. Umso mehr Bedeutung erhalten wiederum die Unterstützungsmöglichkeiten in der Familie und somit die soziale Herkunft.

Die gegenwärtige Krise zeigt auch sehr deutlich, dass es mit einer kurzfristigen Digitalisierung von Schule nicht getan sein kann, nach dem Motto: „Es geht doch irgendwie“. Eine konsequente Berücksichtigung sozialer Ungleichheiten wäre für anstehende schulpolitische Entscheidungen aus den genannten Gründen wirklich wichtig. Unsere Gesellschaft leistet sich nicht ohne Grund mit erheblichem Aufwand ein Schulsystem, das eigentlich Primärerfahrungen im sozialen Kontext ermöglicht. In diesen Zeiten wird besonders deutlich, was wir ohne Schule in Präsenz aufgeben. Und die Corona-Krise hält dem Bildungssystem derzeit den Spiegel vor: Bekannte, aber gerne ignorierte Baustellen werden sichtbar, ohne dass man daran vorbeischauen könnte.

Natürlich muss es auch mittelfristig um die Verlängerung bzw. Erweiterung des DigitalPakt Schule gehen, um die technische und pädagogische Unterstützung der Lehrkräfte im Bereich der Digitalisierung zu gewährleisten, und auch die Lehrerbildung ist sehr gefordert und muss den Erwerb medienpädagogischer Kompetenzen noch viel stärker in die Ausbildung integrieren, um der digitalen Spaltung entgegenzuwirken. Vieles wird im Zuge der aktuellen Situation schneller erfolgen, getragen von einem enormen Engagement vieler Akteure im Bildungsbereich, vor allem auch in der Praxis.

\section{Die Chance in der Krise für die benachteiligten Kinder}

Was aber ganz kurzfristig notwendig und auch machbar erscheint: Kinder aus weniger privilegierten Herkunftsfamilien in Kleingruppen mit individuellen Fördermöglichkeiten zuerst den Zugang in die Schule als Lern- und Lebensort zu ermöglichen, der sie entlasten und stärken kann. Die Schulen können sicherlich beurteilen, 
welche Kinder und Jugendlichen hier zuvorderst $\mathrm{zu}$ adressieren sind. Warum nicht endlich das nachvollziehen, was alle Studien belegen und bereits viele Bildungsakteure derzeit fordern, nämlich besonders benachteiligten Schülerinnen - natürlich unter Berücksichtigung des Gesundheitsschutzes - eine intensive Betreuung zu geben und damit eine Chance? Dabei können auch Lehramtsstudierende unterstützen, am besten begleitet durch die Hochschulen, mit Verträgen oder auch Anrechnungen von Praxisphasen. Die Corona-Krise bietet hier Möglichkeiten für unkonventionelles Handeln und eine Chance für mehr Bildungsgerechtigkeit in einer (nicht nur) digitalen Welt, in der Zugang zu Bildung vor Ort und über digitale Infrastruktur (etwa durch Ausleihmöglichkeiten für Laptops und Tablets) systematisch hergestellt wird.

Isabell van Ackeren, Prof. Dr., Professorin für Bildungssystem- und Schulentwicklungsforschung an der Universität Duisburg-Essen und dort Prorektorin für Studium und Lehre. Sie ist eine der Erstunterzeichner*innen eines offenen Briefs an die KMK zur bevorzugten Öffnung von Schule für sozial benachteiligte Kinder und Jugendliche (https://deutsches-schulportal.de/content/uploads/2020/04/Offener-Brief-an-dieKMK.pdf).

E-Mail: isabell.van-ackeren@uni-due.de

Manuela Endberg, Dr., Leiterin des Forschungsbereichs „Schulentwicklung und Digitalisierung“ in der Arbeitsgruppe Bildungsforschung an der Universität Duisburg-Essen.

E-Mail: manuela.endberg@uni-due.de

Oliver Locker-Grütjen, Dr. (Bildungswissenschaften), Präsident der Hochschule Rhein-Waal.

E-Mail: praesident@hochschule-rhein-waal.de

Korrespondenzadresse: Universität Duisburg-Essen, Arbeitsgruppe Bildungsforschung, Universitätsstr. 2, 45141 Essen 